\title{
Parallel gas spectroscopy using mid-infrared supercontinuum from a single $\mathrm{Si}_{3} \mathbf{N}_{\mathbf{4}}$ waveguide
}

\author{
Eirini Tagkoudi, ${ }^{1}$ (i) Davide Grassani, ${ }^{1,4}$ Fan Yang, ${ }^{2}$ Clemens Herkommer, ${ }^{3}$ (i) \\ Tobias Kippenberg, ${ }^{3}$ and Camille-Sophie Brès ${ }^{1, *}$ \\ ${ }^{1}$ Ecole Polytechnique Fédérale de Lausanne (EPFL), Photonic Systems Laboratory (PHOSL), Lausanne CH-1015, Switzerland \\ ${ }^{2}$ Ecole Polytechnique Fédérale de Lausanne (EPFL), Group for Fiber Optics (GFO), Lausanne CH-1015, Switzerland \\ ${ }^{3}$ Ecole Polytechnique Fédérale de Lausanne (EPFL), Laboratory of Photonics and Quantum measurement (K-Lab), Lausanne CH-1015, \\ Switzerland \\ ${ }^{4}$ Current address: Dipartimento di Fisica, Università degli Studi di Pavia, via Bassi 6, 27100 Pavia, Italy \\ ${ }^{*}$ Corresponding author: camille.bres@epfl.ch
}

Received 7 February 2020; accepted 1 March 2020; posted 6 March 2020 (Doc. ID 390086); published 7 April 2020

Efficient third-order nonlinear optical processes have been successfully integrated on silicon nitride $\left(\mathrm{Si}_{3} \mathrm{~N}_{4}\right)$ waveguides. In particular, owing to $\mathrm{Si}_{3} \mathrm{~N}_{4}$ wide transparency window spanning from the visible to the middle-infrared (mid-IR), efficient mid-IR dispersive-wave (DW) generation from a fiber laser has been recently demonstrated, and its potential as a source for absorption spectroscopy of a single gas has been established. Here we show that the system can be further engineered to broaden the coverage of a single DW without losing efficiency, as to enable simultaneous and discrete detection of several gas-phase molecules within the 2900 and $3380 \mathrm{~cm}^{-1}$ functional group region. We demonstrate quantitative detection of acetylene, methane, and ethane using a simple direct-absorption spectroscopy scheme, achieving a several hundreds of partsper-million noise-equivalent detection limit with a $5 \mathrm{~cm}$ long gas cell. () 2020 Optical Society of America

https://doi.org/10.1364/OL.390086

Laser spectroscopy has been a well-established and powerful technique for resolving molecular linewidths since the early $1980 \mathrm{~s}$ [1]. In particular, middle-infrared (mid-IR) gas spectroscopy has attracted significant interest throughout the past decades due to the presence of strong absorption fingerprints of trace-gases in this spectral region and to the importance of their detection and analysis in environmental monitoring, safety applications, and breath analysis associated with early-stage medical diagnosis [2-4]. Especially, the $\mathrm{C}-\mathrm{H}$ fundamental vibrational transition bands that lie in the spectral range between 3 and $4 \mu \mathrm{m}$ need to be accurately resolved, since they are present in many molecules and are used for the detection of methane $\left(\mathrm{CH}_{4}\right)$, ethane $\left(\mathrm{C}_{2} \mathrm{H}_{6}\right)$, acetylene $\left(\mathrm{C}_{2} \mathrm{H}_{2}\right)$, and a huge number of hydrocarbons [4]. $\mathrm{CH}_{4}$ and $\mathrm{C}_{2} \mathrm{H}_{6}$ are important greenhouse gases, and their quantification is crucial for environmental monitoring [5], while $\mathrm{C}_{2} \mathrm{H}_{2}$ can be hazardous to the industrial production safety [6]. Therefore, it is important to develop compact and simple-operation devices for simultaneous detection of multiple gas-phase species in the mid-IR.
Quantum cascade lasers (QCLs) and interband cascade lasers (ICLs) are currently the most widely used laser sources for precise mid-IR molecular spectroscopy $[7,8]$. An efficient alternative to these tools, which can provide extra tunability, is the generation of mid-IR light through nonlinear conversion. Mid-IR radiation used for spectroscopic applications can be generated through numerous techniques such as optical parametric oscillation (OPO) and difference frequency generation (DFG) [3]. Frequency conversion based on on-chip nanophotonic waveguides offers many advantages compared with nonlinear optical fiber platforms owing to their compactness, lithographic control of their dimensions leading to sensitive engineering of their dispersion profiles, higher nonlinearity, and ultrabroadband transparency windows from the visible to the mid-IR depending on the material [9-11]. However, it has been difficult to reach the mid-IR by direct fiber-laser pumping. Recently, improvements in fabrication processes [12] allowed advanced dispersion engineering for the generation of mid-IR dispersive waves (DWs) in silicon nitride $\left(\mathrm{Si}_{3} \mathrm{~N}_{4}\right)$ waveguides pumped with an erbium-doped fiber laser at $1.55 \mu \mathrm{m}$, which was a significant step towards compact mid-IR frequency comb sources [13]. Nevertheless, limitations in terms of conversion efficiency (CE) beyond $3 \mu \mathrm{m}$ implies necessary input average power levels of hundreds of milliwatts in order to reach the milliwatt ( $\mathrm{mW}$ )-level mid-IR power range required for spectroscopic application [13]. We recently demonstrated that $\mathrm{CE}$ can be significantly enhanced by redshifting the pump wavelength to the thulium band while maintaining the desired phase-matching conditions, reaching power levels now suited for absorption spectroscopy [14]. However, from a given DW, we could only detect a single gas because of a limited DW spectral width. While the detection of several gas species could be achieved in theory from different DWs, this would require switching between different waveguides with varying dimensions or multiplexing of waveguide outputs.

Here we provide a simple alternative for detection of multiple trace-gases from a single fiber-laser and a single straight waveguide. We show that owing to the combination of pump 
wavelength fine-tuning and improved conversion efficiency, we can significantly broaden the spectral width of a DW centered at a specific wavelength for successful simultaneous detection of different gases based on the $\mathrm{C}-\mathrm{H}$ fundamental transition lines. We leverage the broadband generation of the DW-based mid-IR source for direct absorption spectroscopy of $\mathrm{C}_{2} \mathrm{H}_{2}, \mathrm{CH}_{4}$, and $\mathrm{C}_{2} \mathrm{H}_{6}$ spanning the entire 3 to $3.5 \mu \mathrm{m}$ spectral window. We compare the experimental results with a HITRAN database and carry out a study of the signal-to-noise (SNR) ratio for different numbers of averages in the detector, confirming that the high efficiency of the source provides a clear advantage.

The mid-IR DW is obtained from dispersion engineered $\mathrm{Si}_{3} \mathrm{~N}_{4}$ waveguides pumped with an off-the-shelf femtosecond thulium-doped fiber laser (Brevity $\lambda+$, NOVAE) which outputs $90 \mathrm{fs}$ pulses at a $20 \mathrm{MHz}$ repetition rate [Fig. 1(a)], and with a wavelength tunability between 2.07 and $2.1 \mu \mathrm{m}$ [see Fig. 1(b)]. The waveguides consist of a straight $\mathrm{Si}_{3} \mathrm{~N}_{4}$ core buried in a silicon dioxide $\left(\mathrm{SiO}_{2}\right)$ cladding, with a $5 \mathrm{~mm}$ total length, fabricated with the photonic Damascene process [12]. We estimate the total losses as $\sim 11 \mathrm{~dB}$ for the TM fundamental mode, while the propagation losses are $0.2 \mathrm{~dB} / \mathrm{cm}$. Depending on the waveguide cross section, the DW can be generated between 3

(a)

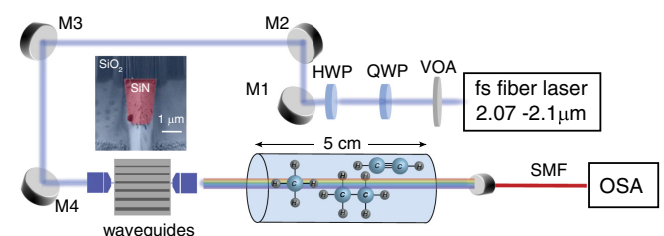

(b)

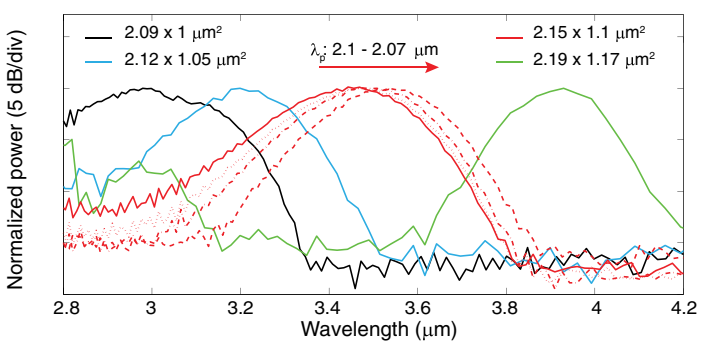

(c)

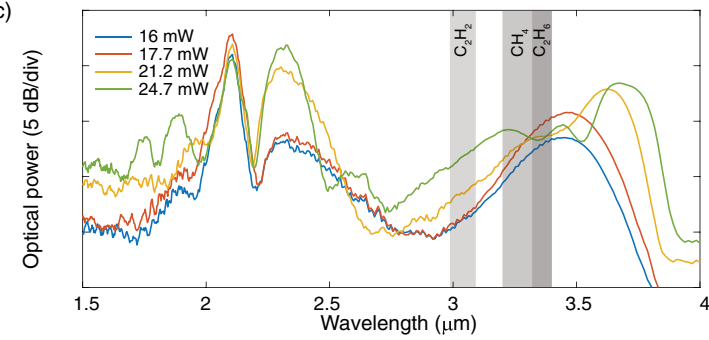

Fig. 1. (a) Experimental setup. M, mirror; HWP, half-wave plate; QWP, quarter-wave plate; VOA, variable optical attenuator. The scanning electron microscopic (SEM) picture of the waveguide cross section is also shown. (b) Tunability of DWG based on waveguides with different cross sections (spectra are normalized), $20 \mathrm{~mW}$ average coupled power. Fine tunability obtained through pump wavelength $\left(\lambda_{p}\right)$ tuning, between 2.07 and $2.1 \mu \mathrm{m}$. (c) Experimentally observed supercontinuum generation in a $2.15 \times 1.1 \mu \mathrm{m}^{2}$ waveguide pumped at $2.1 \mu \mathrm{m}$ with average coupled powers from 16 to $24.7 \mathrm{~mW}$. Spectral region for spectroscopy shown in gray: the $\mathrm{C}_{2} \mathrm{H}_{2}$ absorption lines are in the 2.95-3.1 $\mu \mathrm{m}$ range, the $\mathrm{CH}_{4}$ main absorption lines lie in the $3.2-3.32 \mu \mathrm{m}$ range, and the $\mathrm{C}_{2} \mathrm{H}_{6}$ lines lie in the $3.32-3.36 \mu \mathrm{m}$ range. Resolution: $0.1 \mathrm{~nm}$ (FT-OSA).
Table 1. Experimental Values from the $2.15 \times 1.1 \mu \mathrm{m}^{2}$ Waveguide

\begin{tabular}{lcccc}
$\begin{array}{l}\text { Coupled Pump } \\
\text { power (mW) }\end{array}$ & 24.7 & 21.2 & 17.7 & 16 \\
$\begin{array}{l}\text { On-chip DW } \\
\text { power (mW) }\end{array}$ & 8.4 & 6 & 4 & 3.8 \\
$\begin{array}{l}\text { Output DW power } \\
\text { (mW) }\end{array}$ & 2.4 & 1.7 & 1.13 & 1.07 \\
$\begin{array}{l}\text { On-chip CE (\%) } \\
\text { DW bandwidth }\end{array}$ & 34 & 28.4 & 22.6 & 23.75 \\
$(\mathbf{n m})(\mathbf{a t}-\mathbf{1 0 ~ d B})$ & 1000 & 820 & 750 & 750 \\
\hline
\end{tabular}

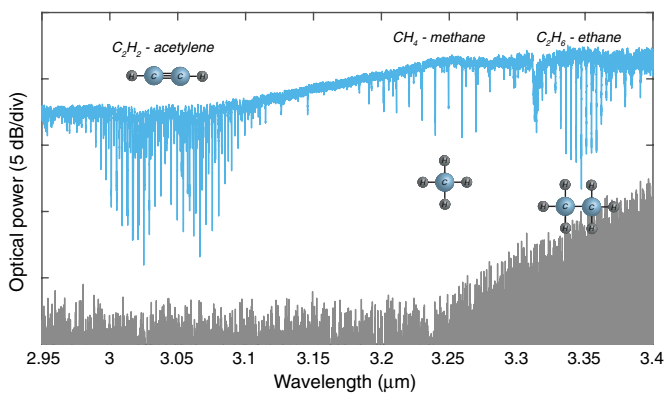

Fig. 2. Transmission spectrum at the output of the gas cell (cyan) observed on the OSA. The noise level is shown in gray. Resolution $0.1 \mathrm{~nm}$.

and $4 \mu \mathrm{m}$, as shown in Fig. 1 (b). The $2.15 \times 1.10 \mu \mathrm{m}^{2}$ waveguide is chosen for the multi-gas spectroscopy experiment. Using this waveguide, the generated DW centered at $3.5 \mu \mathrm{m}$ offers the best overlap with $\mathrm{CH}_{4}$ and $\mathrm{C}_{2} \mathrm{H}_{6}$ absorption lines. Additionally, we can finely tune the DW, so as to provide the optimized performance given the targeted gas, by varying the pump wavelength in a specific waveguide. In particular, one edge of the DW can be favored by 5 to $10 \mathrm{~dB}$ with respect to the other [Fig. 1(b)]. In order to cover the absorption lines of $\mathrm{C}_{2} \mathrm{H}_{2}$, we positioned the pump at $2.1 \mu \mathrm{m}$. The evolution of the spectra at the output of the chip is shown in Fig. 1(c). We observe that the total power in the DW, the CE, and then the DW bandwidth increase with pump power. As such, the DW obtained with $24.7 \mathrm{~mW}$ of coupled pump power covers from 2.86 to $3.86 \mu \mathrm{m}$. This large broadening provides coverage of the three targeted gases, without having to switch to a waveguide with smaller dimensions such as $2.09 \times 1 \mu \mathrm{m}^{2}$ or $2.12 \times 1.05 \mu \mathrm{m}^{2}$ [14]. The experimental values of the mid-IR source are shown in Table 1 .

Light from the output of the waveguide is directly coupled into a short gas cell filled with the three targeted gases $\left(\mathrm{C}_{2} \mathrm{H}_{2}\right.$, $\mathrm{CH}_{4}$, and $\mathrm{C}_{2} \mathrm{H}_{6}$ ). The gas cell has a total path length of $5 \mathrm{~cm}$ with $\mathrm{MgF}_{2}$ optics in the windows. After the interrogation of the gas sample with the DW source, the collimated mid-IR light is focused using a parabolic mirror collimator into a single-mode indium fluoride $\left(\mathrm{SMF}_{\mathrm{InF}_{3}}\right)$ fiber and the spectra are recorded with an optical spectrum analyzer (OSA Yokogawa AQ6376, wavelength reach of $3.4 \mu \mathrm{m}$ ). Figure 2 shows the overall transmission spectrum collected after the gas cell. The maximum dynamic range of the gas absorption measurement is $15 \mathrm{~dB}$ and decreases close to the OSA's detection edge due to an increase in the noise floor (gray curve). 

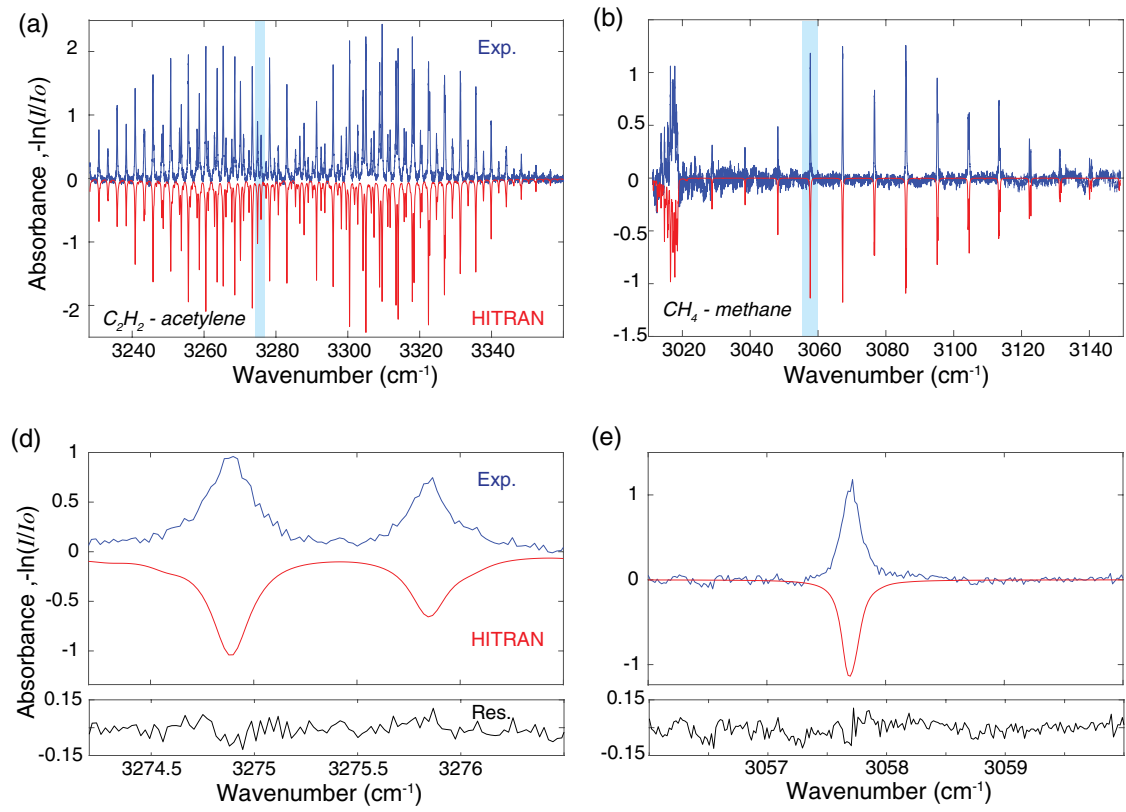

(e)

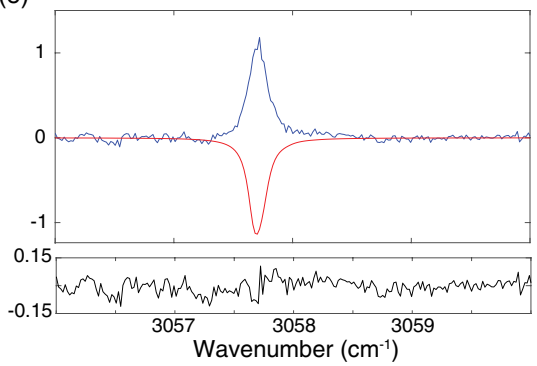

(c)

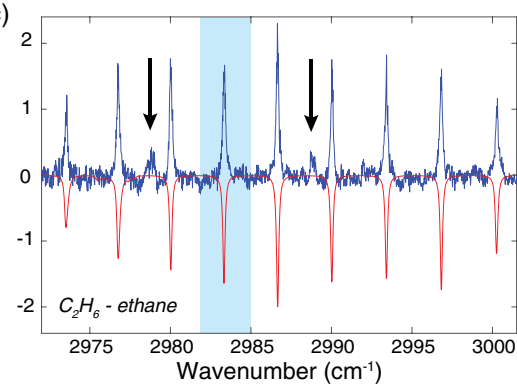

(f)

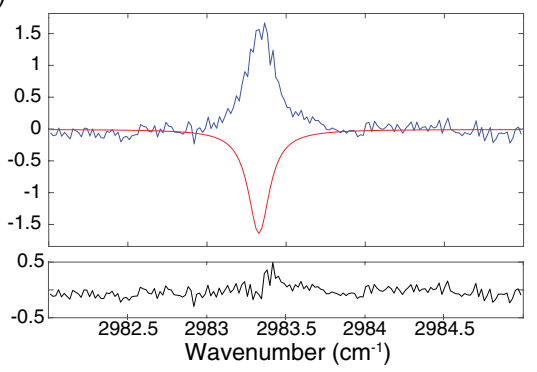

Fig. 3. Normalized experimental gas absorbance (blue curve) and the HITRAN database (red curve, inverted) for (a) $\mathrm{C}_{2} \mathrm{H}_{2},(\mathrm{~b}) \mathrm{CH}_{4}$, and (c) $\mathrm{C}_{2} \mathrm{H}_{6}$. The light blue shaded areas represent the selected for the zoom-in graphs shown in (d) for $\mathrm{C}_{2} \mathrm{H}_{2}$, (e) $\mathrm{CH}_{4}$, and (f) $\mathrm{C}_{2} \mathrm{H}_{6}$, together with the residual (black line). In (c), the black arrows indicate two of the $\mathrm{CH}_{4}$ absorption lines which are also confirmed by HITRAN.

In order to retrieve the normalized spectra, we obtain every measurement with the multi-gas cell and then subtract the reference measurement without the gas cell. The spectral absorbance is then calculated by $-\ln \left(I / I_{o}\right)$, where $I$ is the transmitted spectrum containing the gases, and $I_{o}$ is the reference spectrum. All spectra are recorded with a wavelength resolution of $0.1 \mathrm{~nm}$ using the high sensitivity setting of the internal chopper of the Yokogawa OSA AQ6376 and a sampling step of $0.02 \mathrm{~nm}$. Data acquisition over the $2.95-3.4 \mu \mathrm{m}$ spectral band was performed for 1, 2, 4, and 8 numbers of averages set on the OSA. After removing a remaining slowly varying background with a ninth-order polynomial, we fit our measured absorption spectra to a model based on HITRAN 2016 [15]. The data provided by HITRAN include the spectral line intensity, line central frequency, air-broadening and self-broadening coefficients, and pressure shift coefficient, as well as the isotope peak line strength. Both Doppler and pressure broadening contribute in this case; thus, a Voigt function is applied to simulate separately the line shapes of the 3 gases [16]. The length of the cell, the temperature, as well as the partial and the total pressure of each gas in the mixture are taken into consideration for the simulations. The difference between the normalized measurements and the HITRAN fitted data is plotted as the residual curve for each gas, in which a least-square fitting is applied. The signal-to-noise ratio (SNR) can then be directly extracted from the standard deviation $(\sigma)$ of the baseline noise (residuals) and is given by the relationship $\mathrm{SNR}=\alpha_{\text {peak }} / \sigma$, where $\alpha_{\text {peak }}$ is the peak molecular absorbance.

The normalized experimental spectra and the comparison with the HITRAN simulations (inverted for clarity) are presented in Figs. 3(a)-3(c) for $\mathrm{C}_{2} \mathrm{H}_{2}, \mathrm{CH}_{4}$, and $\mathrm{C}_{2} \mathrm{H}_{6}$, respectively. The correspondence between the experimental data and the HITRAN database is clearly seen in the zoom-in graphs shown in Figs. 3(d)-3(f), with the corresponding residuals plotted in black below. The standard deviations of the residual for the three gases using the spectral ranges shown in Figs. 3(d)$3(\mathrm{f})$ are calculated to be $\sigma=0.045$ for $\mathrm{C}_{2} \mathrm{H}_{2}, \sigma=0.038$ for $\mathrm{CH}_{4}$, and $\sigma=0.11$ for $\mathrm{C}_{2} \mathrm{H}_{6}$. The smallest standard deviation is obtained for $\mathrm{CH}_{4}$, owing to the higher power spectral density of the mid-IR DW in the vicinity of the $\mathrm{CH}_{4}$ absorption lines, as well as a still relatively low noise floor from the OSA. On the other hand, the largest standard deviation for $\mathrm{C}_{2} \mathrm{H}_{6}$ mainly comes from the increased noise level of the OSA at this wavelength range. We can note that lines from $\mathrm{CH}_{4}$ can evidently be seen in Fig. 3(c), as indicated by the black arrows, despite being much weaker than the $\mathrm{C}_{2} \mathrm{H}_{6}$ lines, showing that the two gases can be detected within the same spectral span. Overall, the analysis shows an excellent agreement between the HITRAN model and the experimental results. The mole fractions of $\mathrm{C}_{2} \mathrm{H}_{2}, \mathrm{CH}_{4}$, and $\mathrm{C}_{2} \mathrm{H}_{6}$ are extracted from the fittings of the absorbance spectra [Figs. 3(a)-3(c)]. We obtain $\chi_{\mathrm{CH} 4}=5513 \mathrm{ppm}$ for $\mathrm{CH}_{4}, \chi_{\mathrm{C} 2 \mathrm{H} 2}=19760 \mathrm{ppm}$ for $\mathrm{C}_{2} \mathrm{H}_{2}$, and $\chi_{\mathrm{C} 2 \mathrm{H} 6}=9149 \mathrm{ppm}$ for $\mathrm{C}_{2} \mathrm{H}_{6}$. The noise-equivalent detection limit, defined as $\chi / \mathrm{SNR}_{\max }$, is therefore $160 \mathrm{ppm}$ for $\mathrm{CH}_{4}$, $824 \mathrm{ppm}$ for $\mathrm{C}_{2} \mathrm{H}_{2}$, and $436 \mathrm{ppm}$ for $\mathrm{C}_{2} \mathrm{H}_{6}$. The parts-permillion-level detection limit could be anticipated by using a longer optical path length. A strong point of our mid-IR source comes from the combination of the dispersion engineered $\mathrm{Si}_{3} \mathrm{~N}_{4}$ waveguide and the tuning of the pumping wavelength so as to efficiently generate the mid-IR DW. In the following set of experiments, we investigate how the efficiency of the source impacts the detection performance. We measure the SNR for all three gases for the four different mid-IR DW powers obtained by adjusting the coupled pump power as specified in Table 1 , and different numbers of averages. The calculated SNRs as a function of the number of averages are shown in Figs. 4(a)-4(c). We selected five absorption peaks from different areas of the spectrum for each of the three gases to extract the SNR. The points of Fig. 4 represent the mean value of the SNRs, and error bars have been added based on the extracted standard deviation. 

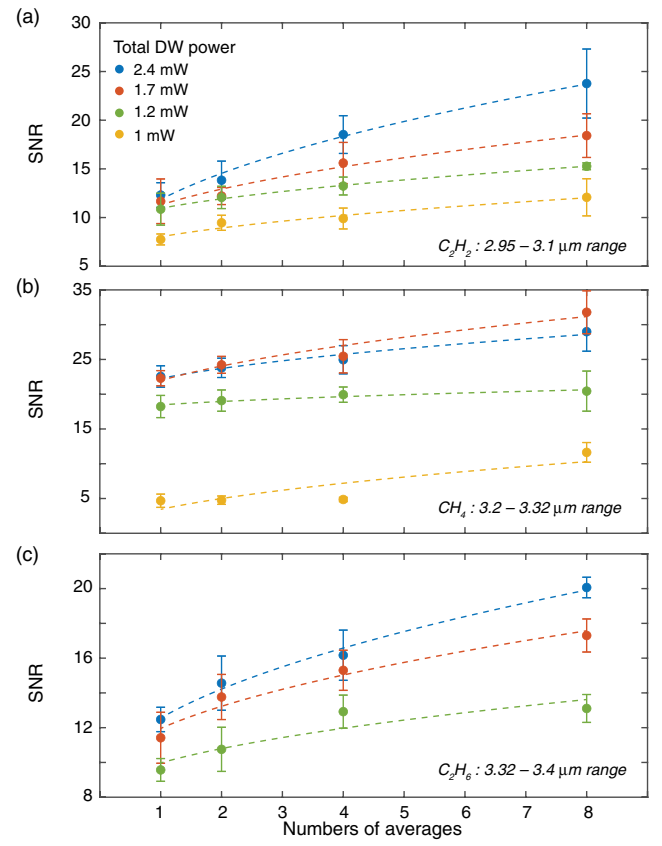

Fig. 4. SNR retrieved from the residuals for four mid-IR DW powers $(2.4,1.7,1.2$, and $1 \mathrm{~mW})$ as a function of the number of averages for (a) $\mathrm{C}_{2} \mathrm{H}_{2}$, (b) $\mathrm{CH}_{4}$, and (c) $\mathrm{C}_{2} \mathrm{H}_{6}$. A square root fitting is applied showing SNR dependence on the number of averages.

The SNR follows a square root dependence on the number of averages, since the system noise is dominated by white noise. A mismatch between the square root fittings and the measured SNRs is attributed to drifts in the DW power in each spectral region during the averaging. As expected, the SNR increases with the number of averages and best SNRs for all three gases are obtained for the highest mid-IR DW power.

The graphs also point to the importance of having an efficient and broad mid-IR generation. In Fig. 4(c), which is the SNR for $\mathrm{C}_{2} \mathrm{H}_{6}$ within the spectral region at the limit of our OSA wavelength range, we observe that the detection is not possible unless a minimum of $1.2 \mathrm{~mW}$ of DW is generated. This is due to the higher noise level of the OSA, which masks the $\mathrm{C}_{2} \mathrm{H}_{6}$ absorption lines at lower powers. For the case of $\mathrm{CH}_{4}$ [Fig. 4(b)], with absorption lines perfectly centered with the DW peak, increasing the total mid-IR DW power up to $1.7 \mathrm{~mW}$ significantly improves the performance and allows for much faster measurements. A further increase of power does not seem to provide an additional advantage within this wavelength range. This is explained by the fact that when the coupled pump power is increased towards the maximum value of $24.7 \mathrm{~mW}$, the main consequence is the spectral broadening of the $\mathrm{DW}$, as seen in Fig. 1(c) and quantified in Table 1. While the total DW power increases, the actual power within the $\mathrm{CH}_{4}$ spectral band does not change significantly. However, more and more light is generated on the blue and red side of the DW, allowing for detection of gases in an extended wavelength range. Finally, in Fig. 4(a), we observe an excellent fit in the case of $\mathrm{C}_{2} \mathrm{H}_{2}$ following the good trade-off between DW overlap and equipment noise.

To summarize, we have experimentally demonstrated the simultaneous detection of multiple gas-phase species using for the first time, to the best of our knowledge, a broadband mid-IR
DW generated in a single dispersion tailored $\mathrm{Si}_{3} \mathrm{~N}_{4}$ nanophotonic waveguide. We targeted the spectral region from 2.95 to $3.4 \mu \mathrm{m}$, an important window for mid-IR greenhouse gas spectroscopy, however, not easily accessible with other tabletop laser sources. In addition, this simple scheme provides a broad spectral coverage, up to $1000 \mathrm{~nm}$, from a single fiber pump and a single waveguide. Our system is capable of achieving simultaneous detection of $\mathrm{C}_{2} \mathrm{H}_{2}, \mathrm{CH}_{4}$, and $\mathrm{C}_{2} \mathrm{H}_{6}$ with a several hundreds of parts-per-million detection limit, using direct absorption spectroscopy with only a $5 \mathrm{~cm}$ long gas cell. The reliability of the device is established by an excellent agreement between measured absorption lines and the HITRAN database.

Funding. European Research Council (ERC-2012StG 306630-MATISSE, ERC-2017-CoG 771647PISSARRO); Schweizerischer Nationalfonds zur Förderung der Wissenschaftlichen Forschung (178895).

Acknowledgment. $\mathrm{Si}_{3} \mathrm{~N}_{4}$ waveguide chips were fabricated in the Center of Micro-nanotechnology (CMI) at EPFL by the K-lab.

Disclosures. The authors declare no conflicts of interests.

\section{REFERENCES}

1. W. Demtröder, Laser Spectroscopy 2: Experimental Techniques, 5th ed. (Springer, 2015).

2. N. Picqué and T. W. Hänsch, Nat. Photonics 13, 146 (2019).

3. M. Vainio and L. Halonen, Phys. Chem. Chem. Phys. 18, 4266 (2016).

4. A. Schliesser, N. Picqué, and T. W. Hänsch, Nat. Photonics 6, 440 (2012).

5. M. L. Smith, E. A. Kort, A. Karion, C. Sweeney, S. C. Herndon, and T. I. Yacovitch, Environ. Sci. Technol. 49, 8158 (2015).

6. Q. He, C. Zheng, H. Liu, Y. Wang, and F. K. Tittel, Proc. SPIE 10111, 1011135 (2017).

7. S. Borri, G. Insero, G. Santambrogio, D. Mazzotti, F. Cappelli, I. Galli, and L. Santamaria, Appl. Phys. B 125, 18 (2019).

8. L. A. Sterczewski, J. Westberg, M. Bagheri, C. Frez, I. Curgaftman, C. L. Canedy, and J. R. Meyer, Opt. Lett. 44, 2113 (2019).

9. A. L. Gaeta, M. Lipson, and T. J. Kippenberg, Nat. Photonics 13, 158 (2019).

10. A. Klenner, A. S. Mayer, A. R. Johnson, K. Luke, M. R. E. Lamont, Y. Okawachi, M. Lipson, A. L. Gaeta, and U. Keller, Opt. Express 24, $11043(2016)$

11. D. Y. Oh, K. Y. Yang, C. Fredrick, G. Ycas, S. A. Diddams, and K. J. Vahala, Nat. Commun. 8, 13922 (2017).

12. M. H. P. Pfeiffer, A. Kordts, V. Brasch, M. Zervas, M. Geiselmann, J. D. Jost, and T. J. Kippenberg, Optica 3, 20 (2016).

13. H. Guo, C. Herkommer, A. Billat, D. Grassani, C. Zhang, M. H. P. Pfeiffer, W. Weng, C. S. Brès, and T. J. Kippenberg, Nat. Photonics 12, 330 (2018).

14. D. Grassani, E. Tagkoudi, H. Guo, C. Herkommer, F. Yang, T. J. Kippenberg, and C.-S. Brès, Nat. Commun. 10, 1553 (2019).

15. I. E. Gordon, L. S. Rothman, C. Hill, R. V. Kochanov, Y. Tan, P. F. Bernath, M. Birk, V. Boudon, A. Campargue, K. V. Chance, B. J. Drouin, J.-M. Flaudi, R. R. Gamache, J. T. Hodges, D. Jacquemart, V. I. Perevalov, A. Perrin, K. P. Shine, M.-A. H. Smith, J. Tennyson, G. C. Toon, H. Tran, V. G. Tyuterev, A. Barbe, A. G. Császár, V. M. Devi, T. Furtenbacher, J. J. Harrison, J.-M. Hartmann, A. Jolly, T. J. Johnson, T. Karmana, I. Kleiner, A. A. Kyuberis, J. Loos, O. M. Lyulin, S. T. Massie, S. N. Mikhailenko, N. Moazzen-Ahmadi, H. S. P. Müller, O. V. Naumenko, A. V. Nikitin, O. L. Polyansky, M. Rey, M. Rotger, S. W. Sharpe, K. Sung, E. Starikova, S. A. Tashkun, J. Vander Auwera, G. Wagner, J. Wilzewski, P. Wcisło, S. Yu, and E. J. Zakb, J. Quant. Spectrosc. Radiat. Transfer 203, 3 (2017).

16. M. Gharavi and S. G. Buckley, Appl. Spectrosc. 58, 468 (2004). 